\title{
Transmuscular Quadratus Lumborum Block
} versus Suprainguinal Fascia lliaca Block for Hip Arthroplasty: A Randomized, Controlled Pilot Study

\author{
Heba Nassar' \\ Ahmed Hasanin (D) ${ }^{\prime}$ \\ Mahmoud Sewilam' \\ Heba Ahmed' \\ Mohamed Abo-Elsoud ${ }^{2}$ \\ Omar Taalab (iD) ${ }^{2}$ \\ Ashraf Rady \\ Heba Allah Zoheir (D) \\ 'Department of Anesthesia and Critical \\ Care Medicine, Faculty of Medicine, \\ Cairo University, Cairo, Egypt; \\ ${ }^{2}$ Department of Orthopedic Surgery, \\ Cairo University, Cairo, Egypt
}

Background: This study aimed to investigate the analgesic efficacy and motor block profile of single-shot transmuscular quadratus lumborum block (QLB) in comparison with those of suprainguinal fascia iliaca block (FIB) in patients undergoing hip arthroplasty.

Methods: This randomized, double-blinded, controlled trial included adult patients undergoing hip arthroplasty under spinal anesthesia. Patients were allocated to one of two groups according to the regional block received: FIB group $(n=19)$ or QLB group $(n=17)$. Both study groups were compared with regard to the duration of analgesia (primary outcome), block performance time, pain during positioning for spinal anesthesia, total morphine consumption in the first postoperative $24-\mathrm{h}$ period, quadriceps muscle power, and static and dynamic visual analog scale.

Results: Thirty-six patients were included in the final analysis. Both study groups had comparable durations of analgesia. Postoperative visual analog scale (static and dynamic) values were comparable between the two groups in most readings. The block performance time was shorter in the FIB group. The number of patients with pain during positioning for the subarachnoid block was lower in the QLB group. The total morphine requirement during the first $24 \mathrm{~h}$ was marginally lower in the FIB group, whereas the quadriceps motor grade was higher in the FIB group than in the QLB group at $4 \mathrm{~h}$ and $6 \mathrm{~h}$ after surgery.

Conclusion: Both single-shot blocks, namely the suprainguinal FIB and transmuscular QLB, provide effective postoperative analgesia after hip arthroplasty. FIB showed slightly lower 24-h morphine consumption, while QLB showed better quadriceps motor power.

Clinical Trial Registration: The study was registered at clinical trials registry system before enrollment of the first participant (NCT04005326; initial release date, 2 July 2019; https://clinicaltrials.gov/ct2/show/NCT04005326).

Keywords: quadratus lumborum block, fascia iliaca block, hip arthroplasty

\section{Introduction}

Postoperative pain is a serious concern in hip arthroplasty. Several modalities are usually used for postoperative analgesia in patients undergoing hip arthroplasty, including neuraxial analgesia, intravenous analgesia, and peripheral nerve blocks. Peripheral nerve blocks have the advantage of potent analgesia and minimal systemic complications. ${ }^{1}$ Hip arthroplasty is usually performed using a lateral or posterior approach. Proper analgesia of the hip joint requires blockade of the
Correspondence: Ahmed Hasanin Department of Anesthesia and Critical Care Medicine, Faculty of Medicine, Cairo University, 0I Elsarayah Street, Elmanyal, Cairo, II559, Egypt

Tel +20 1095076954

Email ahmedmohamedhasanin@gmail. com 
sensory branches of the lumbar plexus. ${ }^{2}$ The literature supports the analgesic efficacy of some selective regional techniques such as the lumbar plexus block, fascia iliaca block (FIB), and pericapsular injection; however, currently there is no consensus on the superiority of any single technique or combination of techniques for analgesia in hip arthroplasty surgeries. ${ }^{3}$ Suprainguinal FIB involves a recently developed anterior approach to the lumbar plexus, which can be performed using either the landmark technique or the ultrasound-guided technique. Compared to the lumbar plexus block, the FIB was recently reported to be easier and faster to perform. Furthermore, according to some reports, the FIB produced a better analgesic profile than that of the lumbar plexus block in hip arthroplasty surgery. ${ }^{4,5}$

The quadratus lumborum block (QLB) is a relatively new regional block that has performed favorably in lower abdominal surgery. ${ }^{6}$ The QLB encompasses four distinct blocks according to the position of the needle tip in relation to the quadratus lumborum muscle: lateral, posterior, anterior (transmuscular), or intramuscular. ${ }^{7,8}$ Cadaveric studies of the transmuscular QLB showed direct dye spread to the roots and branches of the lumbar plexus; ${ }^{9,10}$ thus, these studies supported the possible benefit of QLB in hip surgery. Few clinical reports have evaluated the use of transmuscular QLB in hip arthroplasty. ${ }^{11,12}$ However, randomized controlled evaluation of the block in comparison with other anatomical blocks is lacking. We hypothesized that the transmuscular approach to QLB is superior to the FIB due to the possible blockade of the lumbar nerve roots in addition to blockade of the branches of the lumbar plexus in the QLB, as opposed to blocking only the branches of the lumbar plexus in FIB. This study aimed to investigate the analgesic efficacy and motor block profile of a single-shot transmuscular QLB in comparison with those of a suprainguinal FIB in patients undergoing hip arthroplasty.

\section{Materials and Methods}

This randomized, double-blind, controlled study was conducted at the Cairo University Hospital from August 2019 to March 2020 after receiving approval (N-144-2018) from the Research Ethics Committee, Faculty of Medicine, Cairo University. Written informed consent was obtained from all participants before enrollment. The study was registered in the clinical trials registry system before enrollment of the first participant (NCT04005326; initial release date, July 2, 2019; https://clinicaltrials.gov/ ct2/show/NCT04005326).

Thirty-eight patients aged 18-70 years, with American Society of Anesthesiologists physical status I-III, scheduled for hip replacement surgeries under subarachnoid block, were included in the study. The exclusion criteria were coagulopathy, infection at the injection site, allergy to local anesthetics, severe cardiopulmonary disease, neuropathies, opioid use for chronic analgesic therapy, contraindication to spinal anesthesia, and inability to comprehend the visual analog scale (VAS).

An online randomization program (http://www.rando mizer.org) was used by a research assistant to generate a random sequence, and each code was enclosed in a sealed opaque envelope. Another research assistant, who was not involved in the outcome assessment, was responsible for opening the envelope. Patients were allocated to either the FIB group or the QLB group. The patients and outcome assessors were blinded to the study group allocation.

Patients were instructed how to represent their level of pain using the VAS, which ranged from 0 to 10 points $(0$ indicating no pain and 10 points indicating the worst pain imaginable).

Upon arrival to the regional anesthesia room, an $18-\mathrm{G}$ peripheral intravenous catheter was inserted, and the patient received intravenous ondansetron $(4 \mathrm{mg})$ and dexamethasone ( $8 \mathrm{mg}$ ). Monitoring included electrocardiography, non-invasive arterial blood pressure, and pulse oximetry.

\section{Block Technique}

Under complete aseptic precautions, an ultrasound machine (ACUSON Freestyle, Siemens Medical Solutions, Inc. USA) was used with a high-frequency linear transducer $(6-11 \mathrm{MHz}$ ) (for FIB) or a lowfrequency curvilinear transducer $(2-5 \mathrm{MHz}$ ) (for QLB).

\section{Technique for Suprainguinal FIB}

In the supine position, the high-frequency linear transducer was placed in the sagittal plane to identify the anterior superior iliac spine, and the transducer was moved medially to identify the fascia iliaca, iliopsoas, sartorius, and internal oblique muscles. Using an in-plane approach, a 100-mm, 21-G block needle tip was positioned deep in the fascia iliaca and above the iliacus muscle. After negative aspiration, $30 \mathrm{~mL}$ of $0.25 \%$ bupivacaine was injected 
incrementally under the fascial plane, aspirating every $5 \mathrm{~mL},{ }^{13}$ (Supplementary Figure S1).

\section{Technique for Transmuscular QLB}

The patient was placed in the lateral position. The curvilinear transducer was placed transversely in the midaxillary line above the iliac crest, and the ultrasound transducer was adjusted posteriorly to show the transverse process of L3 or L4, quadratus lumborum muscle, and psoas major muscle. Then, a $100-\mathrm{mm}, 21-\mathrm{G}$ block needle was inserted in the plane from the posterior edge of the transducer in an anteromedial direction until the ventral fascia of the quadratus lumborum muscle was penetrated. The needle tip was placed between the psoas major and quadratus lumborum muscles. After negative aspiration, $30 \mathrm{~mL}$ of $0.25 \%$ bupivacaine was injected incrementally into the fascial plane ${ }^{14}$ (Supplementary Figure S2). After block completion, the patient was reverted to the supine position.

\section{Subarachnoid Block}

Patients received a subarachnoid block 15 min after the assigned regional block, using a 25-G spinal needle in the lateral position. Fifteen milligrams of hyperbaric bupivacaine and $25 \mu \mathrm{g}$ fentanyl were administered at the L3-L4 or L4-L5 interspace. The subarachnoid block was considered successful when a bilateral block at T12 was achieved. Block success was assessed $10 \mathrm{~min}$ after intrathecal injection by evaluating loss of cold and pin prick sensations (using cold ice and a 23-G needle, respectively), and by loss of motor function of the lower limb. If the subarachnoid block failed, general anesthesia was administered, and the patient was excluded from the study.

\section{Postoperative Pain Management}

Postoperatively, all patients received $1 \mathrm{~g}$ paracetamol every $6 \mathrm{~h}$ and $30 \mathrm{mg}$ ketorolac every $12 \mathrm{~h}$. If the VAS was $\geq 4$, additional intravenous morphine was administered in increments of $2 \mathrm{mg}$ up to a maximum of $4 \mathrm{mg}$ to maintain a resting VAS $\leq 3$.

\section{Assessment of Quadriceps Muscle Power}

Patients were asked to extend their knee while fully flexed in the supine position. The motor block was classified as follows: grade 0 , normal muscle power; grade I, motor weakness; grade II, complete motor paralysis. ${ }^{12}$ Quadriceps muscle power was assessed immediately postoperatively and every $2 \mathrm{~h}$ until complete recovery of the muscle power.

The primary outcome was the duration of analgesia, defined as the time to the first postoperative analgesic request. Other outcomes including postoperative VAS at rest (static) and at movement (dynamic) were evaluated at baseline, $15 \mathrm{~min}$ after block performance, immediately after surgery, and at 2, 4, 6, 8, 12, 18, and 24 $\mathrm{h}$ postoperatively. Pain during positioning for spinal anesthesia, total morphine consumption in the first postoperative $24 \mathrm{~h}$, time needed to perform the block, postoperative quadriceps muscle power, and heart rate and blood pressure at baseline and $15 \mathrm{~min}$ after the block were also evaluated.

All blocks were performed by the same operator and usually for the first round of cases in the list that started at $8 \mathrm{am}$; hence, the 12-h follow-up was usually performed at $10 \mathrm{pm}$ (since the surgery usually lasted for an average of $\sim 2 \mathrm{~h}$ ) and the 18-h follow-up was usually performed at 6 am (which was the 19th postoperative hour).

\section{Sample Size Calculation and Statistical Analysis}

The primary outcome was the duration of analgesia. We performed a pilot study on five patients who underwent hip replacement surgery under spinal anesthesia. In the pilot study, the duration of postoperative analgesia after FIB was $10 \pm 2 \mathrm{~h}$ (unpublished data). We calculated our sample size using MedCalc software to detect a mean difference of $20 \%$ between the two groups $(2 \mathrm{~h})$. A minimum of 34 patients (17 patients per group) was needed to achieve a study power of $80 \%$ and an alpha error of 0.05 . The number of envelopes was increased to 38 (19 envelopes per group) to compensate for possible dropouts.

Categorical data were expressed as frequency (percentage [\%]) and analyzed using the chi-squared test. Normality of continuous data was verified using the Shapiro-Wilk test and data were expressed as mean (standard deviation) or median (quartiles), as appropriate. Continuous data were analyzed using the unpaired $t$-test or Mann-Whitney test as appropriate. KaplanMeier survival curves were constructed to compare the time to the first request for postoperative analgesia between groups. Repeated measures analysis of variance (ANOVA) was used to evaluate block type 
(between-groups factor) and time (repeated measures). The Bonferroni test was used to adjust for multiple comparisons (VAS, muscle power). Statistical significance was set at $P<0.05$. IBM SPSS Statistics for Windows, Version 21.0. (Armonk, NY: IBM Corp), and MedCalc software version 14.10.2 (MedCalc Software bvba, Ostend, Belgium) were used for data analysis.

\section{Results}

Forty-two patients were screened for eligibility. Four patients were excluded because they did not meet our inclusion criteria, and 38 patients were randomized into one of the two groups. Two patients in the QLB group were lost to follow-up due to conversion to general anesthesia; thus, 36 patients were included in the final analysis (Figure 1). Demographic data were comparable

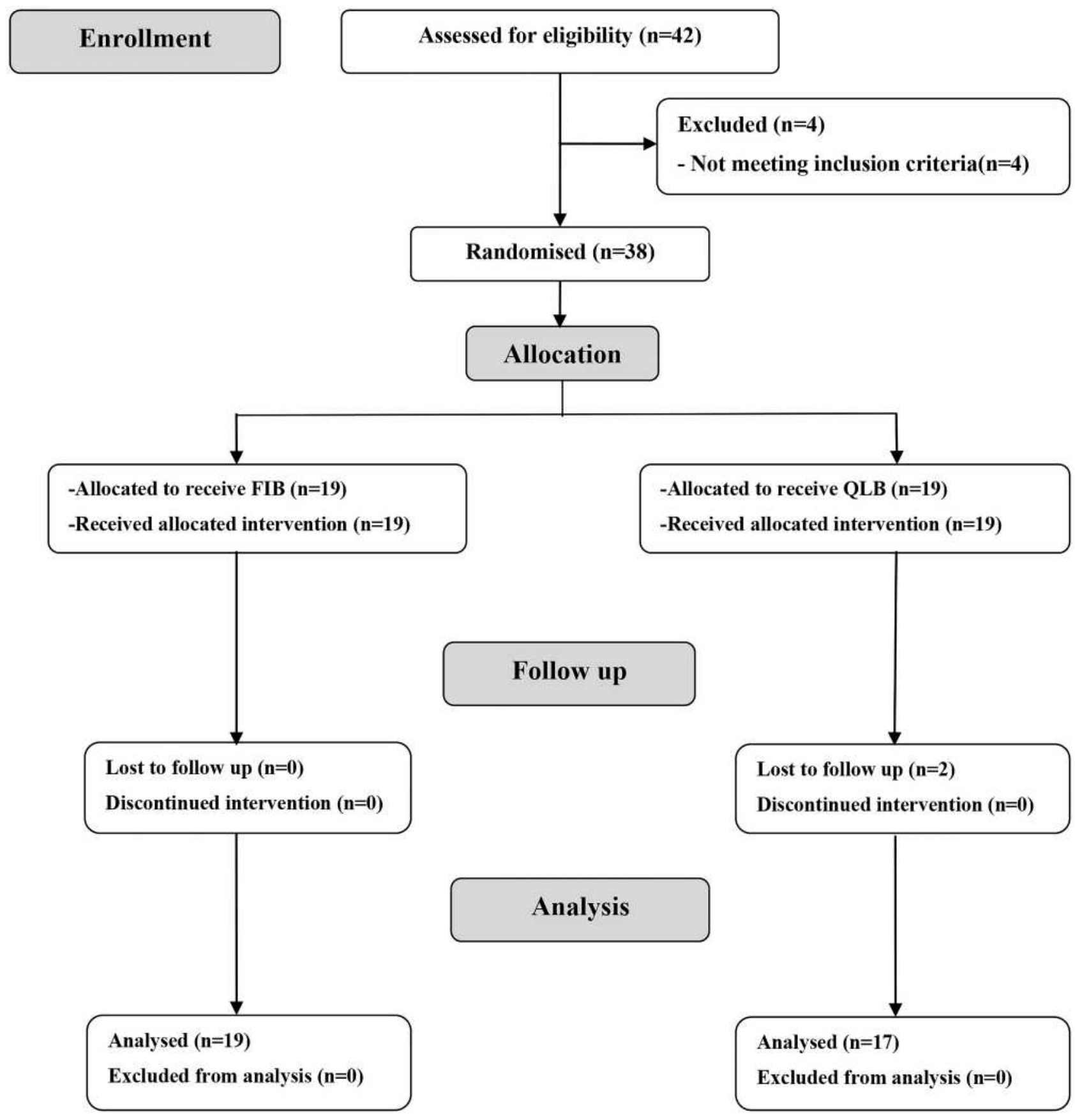

Figure I Flow diagram showing patients' recruitment.

Abbreviations: FIB, fascia iliaca block; QLB, quadratus lumborum block. 
Table I Demographic and Clinical Characteristics

\begin{tabular}{|c|c|c|c|}
\hline Characteristic & $\begin{array}{l}\text { FIB } \\
\text { Group } \\
(n=19)\end{array}$ & $\begin{array}{l}\text { QLB } \\
\text { Group } \\
(n=\mid 7)\end{array}$ & $P$ value \\
\hline Age (years) & $47(17.6)$ & $54(16)$ & 0.195 \\
\hline Male gender, n (\%) & $12(60)$ & $8(40)$ & 0.503 \\
\hline Duration of surgery (min) & $129(23.2)$ & I 34 (2|.5) & 0.494 \\
\hline $\begin{array}{l}\text { Surgery: } \\
\text { Bipolar, n (\%) } \\
\text { Total hip replacement, n (\%) }\end{array}$ & $\begin{array}{l}14(74) \\
5(26)\end{array}$ & $\begin{array}{l}8(47) \\
9(53)\end{array}$ & 0.171 \\
\hline $\begin{array}{l}\text { Patients with pain on } \\
\text { movement before the block, } \\
\mathrm{n}(\%)\end{array}$ & 15 (79\%) & $15(88)$ & 0.662 \\
\hline
\end{tabular}

Note: Data are presented as mean (standard deviation) and frequency (\%). Abbreviations: FIB, fascia iliaca block; QLB, quadratus lumborum block.

between the two groups (Table 1). The indications for hip arthroplasty were mainly severe osteoarthritis (16/19 [84\%] in the FIB group and 14/17 [82\%] in the QLB group) and, to a lesser extent, hip fracture.

The time to the first analgesic request was comparable between the two study groups. The total morphine requirement in the first $24 \mathrm{~h}$ was lower in the FIB group than in the QLB group (median [quartiles]: 6 [4, 8] $\mathrm{mg}$ versus 8 $[7,10] \mathrm{mg}, P=0.049$ ) (Table 2, Figure 2).

The static VAS values were comparable between both groups, while the dynamic VAS values were lower in the QLB group during the first 15 min postblock. Both the static and the dynamic VAS values of

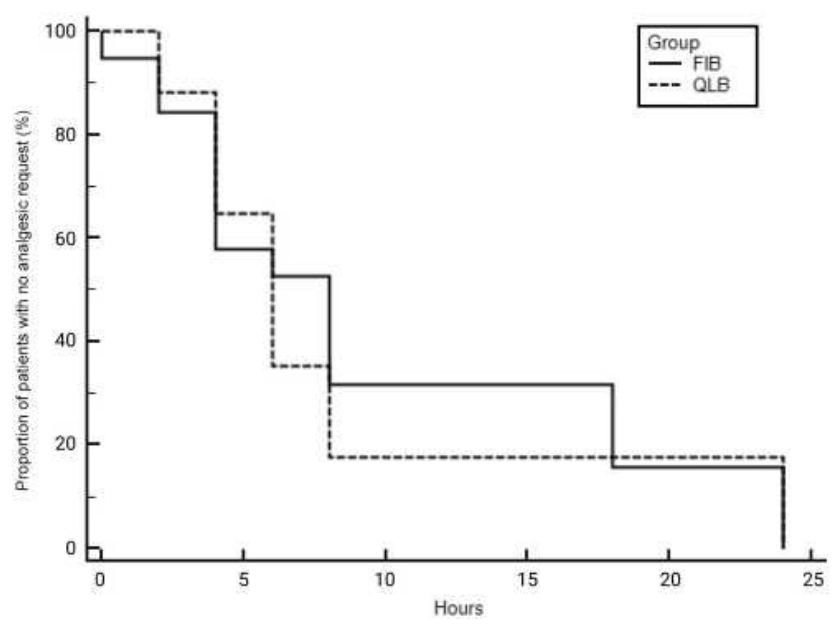

Figure 2 Kaplan-Meier survival analysis for time to first rescue analgesia. Abbreviations: FIB, fascia iliaca block; QLB, quadratus lumborum block.
Table 2 Outcomes and Block Characteristics

\begin{tabular}{|c|c|c|c|}
\hline & $\begin{array}{l}\text { FIB Group } \\
(n=19)\end{array}$ & $\begin{array}{l}\text { QLB } \\
\text { Group } \\
(n=17)\end{array}$ & $P$ value \\
\hline $\begin{array}{l}\text { Block performance time } \\
(\mathrm{sec})\end{array}$ & $60(5)$ & $180(10)^{*}$ & $<0.001$ \\
\hline $\begin{array}{l}\text { Pain during positioning for } \\
\text { spinal anesthesia, n (\%) }\end{array}$ & $10(58 \%)$ & I $(6 \%)^{*}$ & $<0.001$ \\
\hline $\begin{array}{l}\text { Duration of analgesia } \\
\text { (hours) }\end{array}$ & $8[4-18]$ & $6[4-8]$ & 0.420 \\
\hline $\begin{array}{l}\text { Total 24-hour morphine } \\
\text { (mg) }\end{array}$ & $6[4-8]$ & $8[7-10]^{*}$ & 0.049 \\
\hline Baseline heart rate (bpm) & $87(8.5)$ & $95(11.3)^{*}$ & 0.020 \\
\hline $\begin{array}{l}\text { Heart rate (bpm) I5-min } \\
\text { post block }\end{array}$ & $86.9(9.0)$ & $82.4(6.5)$ & 0.110 \\
\hline Baseline SBP $(\mathrm{mmHg})$ & $135(17.7)$ & $134(12)$ & 0.940 \\
\hline $\begin{array}{l}\text { SBP }(\mathrm{mmHg}) \text { I5-min post } \\
\text { block }\end{array}$ & I35.I (16.4) & $118.3(13.1)^{*}$ & 0.002 \\
\hline \multicolumn{4}{|l|}{ Quadriceps power grade } \\
\hline Immediate postoperative & $1.4(0.8)$ & $1.2(0.9)$ & 0.506 \\
\hline 2 hours postoperative & $0.6(0.7)$ & $0.7(0.6)$ & 0.560 \\
\hline 4 hours postoperative & $0.2(0.4)$ & $0(0)^{*}$ & 0.046 \\
\hline 6 hours postoperative & $0.2(0.4)$ & $0(0)^{*}$ & 0.046 \\
\hline 8 hours postoperative & $0.0(0.0)$ & $0(0)$ & 1 \\
\hline 12 hours postoperative & $0.0(0.0)$ & $0(0)$ & 1 \\
\hline
\end{tabular}

Notes: Data are presented as mean (Standard deviation), median [interquartile range], and frequency $(\%)$. *Denotes statistical significance $(P<0.05)$.

Abbreviations: FIB, fascia iliaca block; QLB, quadratus lumborum block.

the two groups were comparable during the first 24 h post-surgery (Tables 3 and 4).

The block performance time was shorter in the FIB group than in the QLB group (60 [5] s vs 180 [10] s, $P<0.001)$. The number of patients who experienced pain during positioning for subarachnoid block was lower in the QLB group than in the FIB group (1 patient $[6 \%]$ versus 10 patients $[58 \%], P<0.001)$ (Table 2).

The quadriceps motor grade was higher in the FIB group than in the QLB group at $4 \mathrm{~h}$ and $6 \mathrm{~h}$ post-surgery and was comparable between the two groups in subsequent measurements (Table 2). The QLB group had lower systolic blood pressure 15 min after block performance than did the FIB group (Table 2). 
Table 3 Static Visual Analogue Scores

\begin{tabular}{|l|l|l|l|}
\hline & $\begin{array}{l}\text { FIB Group } \\
\text { (n=19) }\end{array}$ & $\begin{array}{l}\text { QLB Group } \\
\text { (n=17) }\end{array}$ & P value \\
\hline Baseline & $2.5(1.8)$ & $3(1.5)$ & 0.401 \\
\hline $\begin{array}{l}\text { I5 minutes after } \\
\text { block }\end{array}$ & $1.2(1.3)$ & $0.8(1.4)$ & 0.331 \\
\hline $\begin{array}{l}\text { Immediate } \\
\text { postoperative }\end{array}$ & $0.2(0.9)$ & $0(0)$ & 0.352 \\
\hline $\begin{array}{l}2 \text { hours } \\
\text { postoperative }\end{array}$ & $0.6(1.5)$ & $0.5(1.5)$ & 0.923 \\
\hline $\begin{array}{l}4 \text { hours } \\
\text { postoperative }\end{array}$ & $1.3(1.7)$ & $1.4(1.9)$ & 0.951 \\
\hline $\begin{array}{l}6 \text { hours } \\
\text { postoperative }\end{array}$ & $1.1(1.5)$ & $1.7(1.6)$ & 0.264 \\
\hline $\begin{array}{l}8 \text { hours } \\
\text { postoperative }\end{array}$ & $1.4(1.5)$ & $1.7(1.5)$ & 0.498 \\
\hline $\begin{array}{l}12 \text { hours } \\
\text { postoperative }\end{array}$ & $0.8(1.2)$ & $1.2(1.0)$ & 0.368 \\
\hline $\begin{array}{l}\text { I8 hours } \\
\text { postoperative }\end{array}$ & $1.1(1.3)$ & $0.9(1.0)$ & 0.678 \\
\hline $\begin{array}{l}24 \text { hours } \\
\text { postoperative }\end{array}$ & $1.5(0.9)$ & 0.342 \\
\hline
\end{tabular}

Note: Data are presented as mean (standard deviation).

Abbreviations: FIB, fascia iliaca block; QLB, quadratus lumborum block.

\section{Discussion}

The results of our study revealed that both suprainguinal FIB and transmuscular QLB show comparative postoperative analgesic profiles after hip arthroplasty. We found that both study groups had comparable postoperative pain scores and duration of analgesia, with a moderately lower 24-h opioid consumption in the FIB group. QLB showed two additional advantages: a lower pain score during positioning for subarachnoid block and a more preserved postoperative motor power. The lower VAS in the first reading after block induction in the QLB group, in addition to the lower incidence of pain during positioning, might reflect a faster onset of action compared to the FIB group.

The femoral and obturator nerves are the primary mediators of nociceptive pain in the hip joint. ${ }^{3}$ The site of the cutaneous incision for hip surgery is mainly supplied by the lateral femoral cutaneous nerve. ${ }^{11}$ FIB can provide analgesia after hip surgery by blocking the femoral, obturator, and lateral femoral cutaneous nerves. ${ }^{3}$ The
Table 4 Dynamic Visual Analogue Scores

\begin{tabular}{|l|l|l|l|}
\hline & $\begin{array}{l}\text { FIB Group } \\
\text { (n=19) }\end{array}$ & $\begin{array}{l}\text { QLB Group } \\
\text { (n= I7) }\end{array}$ & P value \\
\hline Baseline & $4.6(1.5)$ & $6.1(1.9)^{*}$ & 0.015 \\
\hline $\begin{array}{l}\text { I5 minutes after } \\
\text { block }\end{array}$ & $4.1(1.4)$ & $2.8(1.8)^{*}$ & 0.031 \\
\hline $\begin{array}{l}\text { Immediate } \\
\text { postoperative }\end{array}$ & $0.3(1.4)$ & $0(0)$ & 0.352 \\
\hline $\begin{array}{l}2 \text { hours } \\
\text { postoperative }\end{array}$ & $1.3(2.3)$ & $1.1(2.7)$ & 0.759 \\
\hline $\begin{array}{l}4 \text { hours } \\
\text { postoperative }\end{array}$ & $2.7(2.5)$ & $2.9(2.6)$ & 0.817 \\
\hline $\begin{array}{l}6 \text { hours } \\
\text { postoperative }\end{array}$ & $2.3(2.3)$ & $3.3(2.4)$ & 0.226 \\
\hline $\begin{array}{l}8 \text { hours } \\
\text { postoperative }\end{array}$ & $3.1(2)$ & $3.8(2.1)$ & 0.301 \\
\hline $\begin{array}{l}12 \text { hours } \\
\text { postoperative }\end{array}$ & $2.8(1.5)$ & $3.1(1.5)$ & 0.603 \\
\hline $\begin{array}{l}18 \text { hours } \\
\text { postoperative }\end{array}$ & $3.2(1.4)$ & $3.2(1.1)$ & 0.953 \\
\hline $\begin{array}{l}24 \text { hours } \\
\text { postoperative }\end{array}$ & $3.8(1.2)$ & 0.368 \\
\hline Notes Dat are & & \\
\hline
\end{tabular}

Notes: Data are presented as mean (standard deviation). *Denotes statistical significance $(P<0.05)$.

Abbreviations: FIB, fascia iliaca block; QLB, quadratus lumborum block.

transmuscular QLB is a relatively new block. The precise mechanism by which the QLB provides analgesia to the hip joint is not fully understood. Several mechanisms can explain the analgesic effect of QLB in our patients. These include (1) medial spread of the local anesthetic to the thoracic and the lumbar paravertebral spaces; ${ }^{14}$ (2) lateral spread to the branches of the lumbar plexus; ${ }^{14}$ and (3) the potential spread to the lumbar plexus via the fascial layer between the anterior two-thirds and the posterior one-third of the psoas muscle. ${ }^{11}$ Cadaveric studies showed direct dye spread to the roots and branches of the lumbar plexus (at levels T10-L3) after transmuscular QLB. ${ }^{9,10}$ However, two randomized controlled clinical trials have shown conflicting results. ${ }^{11,12}$ Kukreja et al reported that QLB provides effective analgesia after hip arthroplasty, ${ }^{11}$ whereas Aoyama et $\mathrm{al}^{12}$ could not find consistent sensory blockade in the lumbar nerves after transmuscular QLB using the same procedure. Our study design differed from those used in these two randomized controlled trials. Kukreja et al compared QLB using a control (no block) regimen, ${ }^{11}$ 
Aoyama et al compared continuous QLB and femoral nerve block, ${ }^{12}$ while our study compared QLB and FIB using a single-shot technique. Furthermore, epinephrine was used as an adjuvant to the local anesthetic mixture for both blocks.

In the present study, postoperative morphine consumption was marginally lower in the FIB group than in the QLB group. This might be hypothetically explained by the blockade of L2-3 dermatomes, especially the lateral femoral cutaneous nerve, in the FIB, which represents a key cutaneous sensation for hip surgery incisions, whereas L3 is not consistently blocked in the QLB. ${ }^{15}$

We found that the FIB was associated with more profound weakness of the quadriceps muscle compared to the QLB. This is most likely due to the usual sparing of the lower lumbar roots in the QLB. ${ }^{10,15}$ On the other hand, quadriceps weakness is a common problem after the FIB. ${ }^{16}$ Motor sparing is generally desirable after joint replacement surgery because it promotes early ambulation of the patients, ${ }^{17}$ thus, a better postoperative motor profile is considered an advantage of the QLB over the FIB.

Our findings introduce the transmuscular QLB as an effective route for analgesia in hip arthroplasty, which showed nearly the same analgesic profile as the FIB in addition to greater preservation of muscle strength. The analgesic profile of the QLB can be further increased by the use of adjuvants (eg, dexamethasone and dexmedetomidine) or by the use of continuous blocks.

Our study has some limitations: during the postoperative period, assessment of the postoperative sensory block was not possible at certain dermatomal levels due to the presence of surgical dressing. The confounding effect of the spinal block impaired the assessment of intraoperative analgesia and early postoperative motor power. We compared the two blocks using the same volume of local anesthetic $(30 \mathrm{~mL})$; however, the comparison of the two blocks might require reevaluation after reaching a consensus for the optimum volume of the local anesthetic in the QLB. We compared the two blocks without adding a control group with a noblock regimen. However, both blocks have been previously compared to control groups and showed superior results to a placebo; ${ }^{11,12}$ therefore, we aimed to compare the two blocks to identify the better analgesic regimen for hip arthroplasty. We calculated the sample size based on data from a pilot study of five adult patients undergoing hip replacement surgery under spinal anesthesia (unpublished data). Studies using data derived from pilot studies for sample size calculation are associated with the smallest gap between the expected and observed power. ${ }^{18}$ However, we observed a large variance in the time to first request for analgesia in the FIB group (8 [4-18] hours) compared to that observed in our pilot study (10 \pm 2 hours), which could explain the underpowered primary outcome in our study. Hence, this study may be considered a pilot study, and a larger study is needed to detect differences between the two groups. Finally, our patients received bupivacaine, which is readily available in our hospital; other local anesthetics may have different effects on the analgesic duration and motor power after the QLB.

In conclusion, within the context of multimodal analgesia, both single-shot blocks, namely the suprainguinal FIB and transmuscular QLB, provide comparable postoperative analgesia after hip arthroplasty.

\section{Data Sharing Statement}

The data that support the findings of this study are available from Cairo university hospitals; however, they are not publicly available. Data are however available from the corresponding author (Dr. Ahmed Hasanin, email: ahemdmohamedhasanin@gmail.com) upon reasonable request after permission of Cairo university.

\section{Ethics Approval and Informed Consent}

We conducted this study in accordance with Declaration of Helsinki and the Research Ethics Committee of Cairo University approved the conduction of this study (N-1442018). Written informed consent was obtained from all patients before the enrollment.

\section{Acknowledgment}

We would like to thank Dr. Maha Mostafa (Lecturer of anesthesia and critical care medicine, Cairo University, Cairo, Egypt) who helped in data analysis.

\section{Author Contributions}

All authors contributed to data analysis, drafting or revising the article, have agreed on the journal to which the article will be submitted, gave final approval of the version to be published, and agree to be accountable for all aspects of the work.

\section{Funding}

There is no funding to report. 


\section{Disclosure}

The authors reported no conflicts of interest for this work. This paper and the abstract of this paper have not been presented at a conference.

\section{References}

1. Capdevila X, Macaire P, Dadure C, et al. Continuous psoas compartment block for postoperative analgesia after total hip arthroplasty: new landmarks, technical guidelines, and clinical evaluation. Anesth Analg. 2002;94(6):1606-1613. doi:10.1097/00000539-200206000-00045

2. Rao ARV, Lakshmanan A, Ajith A, Rao SM. Centenarians: hip fractures and peripheral lower limb nerve blocks. Indian $J$ Anaesth. 2010;54(3):268-269. doi:10.4103/0019-5049.65360

3. Bugada D, Bellini V, Lorini LF, Mariano ER. Update on selective regional analgesia for hip surgery patients. Anesthesiol Clin. 2018;36 (3):403-415. doi:10.1016/j.anclin.2018.04.001

4. Bravo D, Layera S, Aliste J, et al. Lumbar plexus block versus suprainguinal fascia iliaca block for total hip arthroplasty: a single-blinded, randomized trial. J Clin Anesth. 2020;66:109907. doi:10.1016/j.jclinane.2020.109907

5. Desmet M, Balocco AL, Van Belleghem V. Fascia iliaca compartment blocks: different techniques and review of the literature. Best Pract Res Clin Anaesthesiol. 2019;33(1):57-66. doi:10.1016/j. bpa.2019.03.004

6. Albrecht E, Chin KJ. Advances in regional anaesthesia and acute pain management: a narrative review. Anaesthesia. 2020;75(S1):e101e110. doi:10.1111/anae.14868

7. Kinjo S, Kolodzie K, Dong K, Zhang AL. The effects of transmuscular quadratus lumborum blocks on postoperative pain in arthroscopic hip surgery: a cohort analysis. J Anesth. 2019;33(4):516-522. doi:10.1007/ s00540-019-02659-9

8. Lu Y, Zhang J, Xu X, et al. Sensory assessment and block duration of transmuscular quadratus lumborum block at L2 versus L4 in volunteers: a randomized controlled trial. Minerva Anestesiol. 2019;85 (12):1273-1280. doi:10.23736/S0375-9393.19.13656-5

9. Carline L, McLeod GA, Lamb C. A cadaver study comparing spread of dye and nerve involvement after three different quadratus lumborum blocks. Br J Anaesth. 2016;117(3):387-394. doi:10.1093/bja/aew224
10. Elsharkawy H, El-Boghdadly K, Barnes TJ, et al. The supra-iliac anterior quadratus lumborum block: a cadaveric study and case series. Can J Anaesth. 2019;66:849-906.

11. Kukreja P, Macbeth L, Sturdivant A, et al. Anterior quadratus lumborum block analgesia for total hip arthroplasty : a randomized, controlled study. Reg Anesth Pain Med. 2019. doi:10.1136/rapm2019-100804

12. Aoyama Y, Sakura S, Abe S, Tadenuma S, Saito Y. Continuous quadratus lumborum block and femoral nerve block for total hip arthroplasty: a randomized study. J Anesth. 2020;34(3):413-420. doi:10.1007/s00540-020-02769-9

13. Desmet M, Vermeylen K, Van Herreweghe I, et al. A longitudinal supra-inguinal fascia iliaca compartment block reduces morphine consumption after total hip arthroplasty. Reg Anesth Pain Med. 2017;42:327-333. doi:10.1097/AAP.0000000000000543

14. Elsharkawy H, El-Boghdadly K, Barrington M. Quadratus lumborum block: anatomical concepts, mechanisms, and techniques. Anesthesiology. 2019;130(2):322-335. doi:10.1097/ ALN.0000000000002524

15. Sondekoppam RV, Ip V, Johnston DF, et al. Ultrasound-guided lateral-medial transmuscular quadratus lumborum block for analgesia following anterior iliac crest bone graft harvesting: a clinical and anatomical study. Can J Anaesth. 2018;65(2):178-187. doi:10.1007/ s12630-017-1021-y

16. Gasanova I, Alexander JC, Estrera K, et al. Ultrasound-guided suprainguinal fascia iliaca compartment block versus periarticular infiltration for pain management after total hip arthroplasty: a randomized controlled trial. Reg Anesth Pain Med. 2019;44 (2):206-211. doi:10.1136/rapm-2018-000016

17. Yayik AM, Cesur S, Ozturk F, Ahiskalioglu A, Celik EC. Continuous quadratus lumborum type 3 block provides effective postoperative analgesia for hip surgery: case report. Braz J Anesthesiol. 2019;69 (2):208-210.

18. Abdulatif M, Mukhtar A, Obayah G. Pitfalls in reporting sample size calculation in randomized controlled trials published in leading anaesthesia journals: a systematic review. Br J Anaesth. 2015;115 (5):699-707. doi:10.1093/bja/aev166
Local and Regional Anesthesia

\section{Publish your work in this journal}

Local and Regional Anesthesia is an international, peer-reviewed, open access journal publishing on the development, pharmacology, delivery and targeting and clinical use of local and regional anesthetics and analgesics. The journal welcomes submitted papers covering original research, basic science, clinical studies, reviews \&

\section{Dovepress}

evaluations, guidelines, expert opinion and commentary, case reports and extended reports. The manuscript management system is completely online and includes a very quick and fair peer-review system, which is all easy to use. Visit http://www.dovepress.com/testimonials. php to read real quotes from published authors. 